\title{
Imaging of Proliferation in Hepatocellular Carcinoma with the In Vivo Marker ${ }^{18}$ F-Fluorothymidine
}

Florian Eckel ${ }^{* 1}$, Ken Herrmann*2, Stefan Schmidt ${ }^{3}$, Claudia Hillerer ${ }^{2}$, Hinrich A. Wieder ${ }^{3}$, Bernd-Joachim Krause ${ }^{2}$, Tibor Schuster ${ }^{4}$, Rupert Langer ${ }^{5}$, Hans-Jürgen Wester ${ }^{2}$, Roland M. Schmid ${ }^{1}$, Markus Schwaiger ${ }^{2}$, and Andreas K. Buck ${ }^{2}$

${ }^{I}$ Department of Internal Medicine II, Technische Universität München, Munich, Germany; ${ }^{2}$ Department of Nuclear Medicine, Technische Universität München, Munich, Germany; ${ }^{3}$ Department of Radiology, Technische Universität München, Munich, Germany; ${ }^{4}$ Department of Medical Statistics, Technische Universität München, Munich, Germany; and ${ }^{5}$ Department of Pathology, Technische Universität München, Munich, Germany

We determined the ability of PET with the thymidine analog $3^{\prime}$-deoxy-3'-18 F-fluorothymidine $\left({ }^{18} \mathrm{~F}-\mathrm{FLT}\right)$ to detect hepatocellular carcinoma (HCC). Methods: In this pilot study, ${ }^{18} \mathrm{~F}-\mathrm{FLT}$ PET was performed in 18 untreated patients with clinically suspected HCC. Routine diagnostic procedures included ultrasound, MRI, or contrast-enhanced spiral CT of the upper gastrointestinal tract in all patients. At 45-60 min after the intravenous injection of approximately $270-340 \mathrm{MBq}$ of ${ }^{18} \mathrm{~F}-\mathrm{FLT}$, emission and transmission scanning was performed with a high-resolution PET scanner. Tracer uptake in the tumor and surrounding liver tissue was evaluated semiquantitatively by calculation of mean and maximum standardized uptake values (SUVS). Results were correlated with those of the conventional imaging methods. Results: A total of 13 of 18 tumors (sensitivity, $72 \%$; 95\% confidence interval [Cl], 47\%-90\%) showed focal ${ }^{18} \mathrm{~F}-\mathrm{FLT}$ uptake higher than surrounding liver activity and were detectable as hot lesions. Five tumors were characterized as photopenic lesions or contained a mixture of hot and cold lesions exhibiting a comparable or lower ${ }^{18} \mathrm{~F}-\mathrm{FLT}$ uptake than the surrounding liver tissue. When all lesions were considered, the mean ${ }^{18} \mathrm{~F}-\mathrm{FLT}$ SUV was 7.8 (range, 2.5-11.1), and the maximum ${ }^{18}$ F-FLT SUV was 9.3 (range, 2.9-14.3). Histology and clinical follow-up revealed $\mathrm{HCC}$ in 16 patients and cholangiocarcinoma in 2 patients. In the subgroup of HCC, the sensitivity for tumor detection was $69 \%(11 / 16 ; 95 \% \mathrm{Cl}, 41 \%-89 \%)$. Correlation analysis demonstrated a significant positive relationship between the proliferation marker MIB-1 and the mean SUV ( $r=0.66, P=$ 0.02). Survival analysis (Cox proportional hazards regression) for initial ${ }^{18} \mathrm{~F}-\mathrm{FLT}$ uptake (mean and maximum SUVs) revealed increased hazard ratios (mean SUV, 1.20; maximum SUV, 1.12), but because of the small number of events, these results were not statistically significant. Conclusion: In this pilot study, HCC tumors showed a mixed uptake pattern for the in vivo proliferation marker ${ }^{18} \mathrm{~F}-\mathrm{FLT}$. A total of $69 \%$ of the HCC lesions showed ${ }^{18} \mathrm{~F}-\mathrm{FLT}$ uptake higher than that of the surrounding liver tissue, whereas the remaining lesions were photopenic or contained a mixture of hot and cold lesions. High initial ${ }^{18} \mathrm{~F}-\mathrm{FLT}$ uptake seems

Received May 6, 2009; revision accepted May 22, 2009.

For correspondence or reprints contact: Ken Herrmann, Department of Nuclear Medicine, Technische Universität München, Ismaninger Strasse 22, D-81675 Munich, Germany.

E-mail: ken.herrmann@tum.de

${ }^{*}$ Contributed equally to this work.

COPYRIGHT ๑) 2009 by the Society of Nuclear Medicine, Inc. to be associated with reduced overall survival and could be an important prognostic factor if this tendency can be confirmed in a larger prospective trial.

Key Words: ${ }^{18} \mathrm{~F}-\mathrm{FLT}$; HCC; proliferation; PET

J Nucl Med 2009; 50:1441-1447

DOI: 10.2967/jnumed.109.065896

$\mathbf{H}_{0}$ epatocellular carcinoma (HCC) is the fourth leading cause of cancer-related death, represents the most common primary hepatic malignancy worldwide, and is showing an increasing incidence in Western countries (1). Most HCCs appear to be associated with risk factors, such as a hepatitis B carrier state, chronic hepatitis $\mathrm{C}$ virus infection, hereditary hemochromatosis, and cirrhosis of almost any cause (2). However, HCC can also occur in patients without known risk factors (3).

The detection of HCC at a very early (Barcelona Clinic Liver Cancer classification [BCLC] stage 0) or early (BCLC stage A) stage is crucial because curative treatment options, such as surgical resection, transplantation, or local ablation therapy, are not applicable in advanced stages (4). So far, the detection and staging of HCC are the domains of conventional imaging modalities, such as ultrasound, CT, and MRI (5). The main challenge of initial imaging is tumor detection in underlying liver cirrhosis. The cirrhotic liver may contain regenerative or dysplastic nodules as well as HCC.

For intermediate-stage HCC (BCLC stage B), arterial embolization or chemoembolization represents an evidencebased treatment based on positive randomized controlled trials and meta-analysis (4). Pathologic analysis of explanted livers demonstrated nearly complete $(90 \%-100 \%)$ tumor necrosis in $65 \%-71 \%$ after arterial chemoembolization (6). In contrast, objective response rates were only about $35 \%$ (range, 16\%-61\%) (7), suggesting some diagnostic incertitude in assessing the response after arterial embolization by means of conventional imaging modalities. 
PET with the glucose analog ${ }^{18} \mathrm{~F}-\mathrm{FDG}$ proved to have satisfactory sensitivity only for the detection of moderately or poorly differentiated HCC; well-differentiated and lowgrade tumors were not detectable $(8,9)$. Dual-tracer PET/ CT approaches with ${ }^{11} \mathrm{C}$-acetate and ${ }^{18} \mathrm{~F}$-FDG proved to be useful for the evaluation of HCC metastasis, although the role of such approaches in the diagnosis of primary HCC remains limited (10). An alternative tracer, the thymidine analog $3^{\prime}$-deoxy- $3^{\prime}-{ }^{18} \mathrm{~F}$-fluorothymidine $\left({ }^{18} \mathrm{~F}\right.$-FLT), is a new radiopharmaceutical for clinical PET; it specifically visualizes proliferating tissues $(11,12)$. In the present study, we aimed to determine the ability of ${ }^{18}$ F-FLT PET to detect $\mathrm{HCC}$ and to evaluate initial ${ }^{18} \mathrm{~F}$-FLT uptake as a rationale for noninvasive treatment monitoring.

\section{MATERIALS AND METHODS}

\section{Patient Population}

Eighteen patients with primary liver cancer suggestive of HCC were included in this prospective study. Diagnoses were histologically proven or based on the guidelines of the American Association for the Study of Liver Diseases (13). Staging procedures included abdominal sonography, MRI, and CT of the chest and abdomen as clinically appropriate. Histologic or cytologic confirmation or exclusion of the diagnosis was performed when possible. Endoscopic ultrasound-guided or CT-guided fine-needle aspiration and biopsy were performed as clinically appropriate. Informed consent was obtained from all patients. Clinical follow-up was performed (mean follow-up, 12.5 mo; median follow-up, 11.5 mo), and no patient was lost to follow-up. So far, 5 patients have died and 13 are still alive.

\section{${ }^{18}$ F-FLT PET}

${ }^{18} \mathrm{~F}-\mathrm{FLT}$ was synthesized as previously described (14). Imaging was performed on a whole-body high-resolution PET scanner (ECAT HR+; Siemens/CTI, Inc.). This scanner simultaneously acquires 47 contiguous slices with a slice thickness of $3.4 \mathrm{~mm}$. The in-plane image resolution of transaxial images was approximately $8 \mathrm{~mm}$ at full width at half maximum, with an axial resolution of approximately $5 \mathrm{~mm}$ at full width at half maximum. Static emission images were acquired $45 \mathrm{~min}$ after the injection of approximately $300 \mathrm{MBq}$ of ${ }^{18} \mathrm{~F}-\mathrm{FLT}$ (range, 270-340 MBq). Emission data were corrected for random coincidences, dead time, and attenuation and reconstructed by filtered backprojection (Hanning filter with a cutoff frequency of 0.4 cycle per bin). The matrix size was $128 \times 128$ pixels, and the pixel size was $4.0 \times 4.0$ $\mathrm{mm}$. The image pixel counts were calibrated to activity concentrations $(\mathrm{Bq} / \mathrm{mL})$ and decay corrected with the time of the tracer injection as a reference.

\section{PET Data Analysis}

All ${ }^{18}$ F-FLT PET scans were evaluated by 2 experienced nuclear medicine physicians who were unaware of the clinical data and the results of other imaging studies. Circular regions of interest with a diameter of $1.5 \mathrm{~cm}$ were placed in the area with the highest tumor activity as described earlier (15) as well as in representative areas of normal liver tissue. Mean standardized uptake values (SUVs) were calculated from each region of interest with the following formula: SUV $=$ [measured activity concentration $(\mathrm{Bq} / \mathrm{g}) \times$ body weight $(\mathrm{g})] /$ injected activity $(\mathrm{Bq})$. For definition of regions of interest and data analysis, we used computer programs developed in the Interactive Data Language (IDL; Research Systems, Inc.) with the Clinical Application Programming Package (CAPP; Siemens/CTI, Inc.) (16).

\section{MRI Techniques}

MRI was performed in 13 patients with a 1.5 -T system (Avanto; Siemens). Sequences included T2-weighted fast spin echo and T1weighted gradient echo. Fat suppression techniques were used in 7 patients. In-phase and opposed-phase gradient-echo sequences were used in 5 patients. T1-weighted imaging was repeated in all patients after intravenous contrast enhancement during the hepatic arterial (20- to 25-s delay) and portal venous (60- to 70-s delay) phases. Delayed-phase imaging (5-10 min after contrast material injection) was performed in 9 patients. All patients received a gadolinium chelate at a dose of $0.1 \mathrm{mmol}$ per kilogram of body weight and then a $20-\mathrm{mL}$ saline flush.

\section{Helical CT Techniques}

Five of the 18 patients underwent abdominal helical CT, which included unenhanced and contrast-enhanced imaging through the whole liver; imaging during the hepatic arterial and portal venous phases was performed $25-35$ and 60-70 s, respectively, after initiation of the intravenous injection of contrast material. In addition, 3 patients also underwent delayed-phase imaging through the liver 5-10 min after initiation of the administration of contrast material. All CT examinations were performed with multi-detector-row scanners (Somatom; Siemens) at 180-250 $\mathrm{mAs}$ and $120 \mathrm{kVp}$. All patients received nonionic intravenous contrast material (iodine at $350 \mathrm{mg} / \mathrm{mL}$ ) administered at a rate of $3-5 \mathrm{~mL} / \mathrm{s}$ and a volume of $120-150 \mathrm{~mL}$ with a mechanical power injector (Medrad). The section thickness and reconstruction interval were $5 \mathrm{~mm}$, and the pitch was adjusted to allow scanning of the complete liver within one breath hold.

\section{Reference Methods for Assessment of Grading and MIB Labeling Index}

Histopathologic diagnosis was made from biopsy material for 9 patients and from resection specimens for 5 patients. Grading was available for 13 tumors: 2 carcinomas were well differentiated (G1), 9 carcinomas were moderately differentiated (G2), and 2 carcinomas were poorly differentiated (G3). In the remaining 4 patients, no specimen could be obtained; therefore, the diagnosis was based on a combination of imaging methods, such as multislice CT, MRI, and ultrasound, which were repeated if necessary. Immunohistochemical staining for the Ki-67 antigen (monoclonal antibody MIB-1; Dako; applied at a dilution of 1:100) was done with an automated stainer (Ventana Benchmark). Antigen retrieval was performed according to the manufacturer's instructions. A total of 2,000 tumor cells were evaluated for nuclear immunoreactivity in the most active areas of the tumors. In 2 cases, all tumor cells were analyzed because the biopsy material contained fewer than 2,000 tumor cells. The MIB-1 labeling index was expressed as the percentage of cells with positive nuclear staining.

\section{Statistical Analysis}

Statistical analyses were performed with SPSS software (version 16.0; SPSS, Inc.). Quantitative values were expressed as mean $\pm \mathrm{SD}$, median, and range. Comparisons of related metric measurements were performed with the Wilcoxon signed rank test, and the Mann-Whitney $U$ test was used to compare quantitative data from 2 independent samples. The Fisher exact test was used for the comparison of frequencies, and Spearman 
correlation coefficients were calculated to quantify bivariate correlations of measurement data. Exact 2-sided 95\% confidence intervals (CIs) were reported for estimates of sensitivity and specificity.

To investigate the overall performance of the tumor-to-liver (T/ L) ratio in the discrimination of tumors with positive and negative PET results, we performed receiver operating characteristic (ROC) analyses. The corresponding areas under the curve and statistically optimal cutoff values for maximizing sensitivity and specificity were reported. Survival analysis was performed as Cox proportional hazards regression. All analyses were performed 2-sided at a $5 \%$ level of significance.

\section{RESULTS}

Eighteen patients were enrolled in this prospective study (17 men and 1 woman; age: mean $\pm \mathrm{SD}, 67 \pm 9 \mathrm{y}$; range, 48-78 y) (Table 1). HCC was histologically proven in 12 patients: in 4 patients after resection and in 8 patients by liver biopsy (one case was suggestive of HCC). Among the remaining 6 patients, one patient presented with primary liver cancer strongly suggestive of HCC; histologic examination after resection of the tumor demonstrated cholangiocarcinoma (CCC). Another patient, who was referred from an external medical center, had histologically diagnosed HCC; however, no other liver disease, risk factor for $\mathrm{HCC}$, or atypical imaging studies were found. A biopsy performed at our institution yielded the diagnosis of CCC. The other 4 patients had HCC diagnosed according to the guidelines of the American Association for the Study of Liver Diseases. They were treated accordingly, and no doubts about the initial diagnosis were raised during follow-up.
Imaging HCC Tumors with ${ }^{18} \mathrm{~F}-\mathrm{FLT}$ PET

Visual Analysis. ${ }^{18}$ F-FLT PET produced high-contrast images of proliferating structures (Fig. 1). In 13 of 18 patients enrolled, malignant tumors showed focal ${ }^{18} \mathrm{~F}$-FLT uptake higher than surrounding liver activity (sensitivity of ${ }^{18}$ F-FLT PET, 72\%; 95\% CI, 47\%-90\%) (Fig. 1). In one of these 13 patients (patient 4), multifocal lesions showed a mixed pattern of increased uptake and decreased uptake that was determined by visual analysis to be positive even though the uptake was lower than that in the surrounding liver tissue. In the subgroup of patients with $\mathrm{HCC}, 5$ of 16 tumors did not show focally increased tracer uptake (Figs. 2 and 3) compared with the surrounding normal liver activity, leading to a sensitivity of $69 \%(11 / 16 ; 95 \%$ CI, 41\%-89\%) (Table 1). In contrast, for the tumors in both patients with $\mathrm{CCC}$, tracer uptake was determined by visual analysis to be positive.

Quantitative Analysis. The mean ${ }^{18} \mathrm{~F}-\mathrm{FLT}$ uptake (SUV) was 7.6 (median, 7.5; range, 2.5-11.1) in all studied HCC tumors $(n=16)$ and 6.3 (median, 5.7; range, 4.4-9.5) in representative liver tissue. The average maximum ${ }^{18} \mathrm{~F}$-FLT uptake in all HCC tumors $(n=16)$ was 9.0 (median, 8.9; range, 2.9-14.3). Focal maximum ${ }^{18}$ F-FLT uptake was significantly higher, at 10.2 (median, 9.1; range, 7.914.3), in the subgroup of HCC tumors determined by visual analysis to have positive PET results than in tumors with negative PET results (mean of maximum uptake, 6.5; median, 7.3; range, 2.9-9.0) $(P=0.04)$. Focal mean ${ }^{18} \mathrm{~F}-$ FLT uptake was also higher in HCC tumors with positive PET results, at 8.4 (median, 7.7; range, 6.5-11.1), than in lesions with negative PET results (mean, 5.8; median, 6.4;

\begin{tabular}{|c|c|c|c|c|c|c|c|c|c|c|c|}
\hline \multirow[b]{2}{*}{ Patient } & \multirow[b]{2}{*}{ Sex } & \multirow[b]{2}{*}{ Age (y) } & \multirow[b]{2}{*}{$\begin{array}{l}\text { Tumor } \\
\text { type }\end{array}$} & \multirow[b]{2}{*}{ Histology } & \multirow[b]{2}{*}{ Grade } & \multirow[b]{2}{*}{$\begin{array}{l}\text { MIB proliferation } \\
\text { index }(\%)\end{array}$} & \multicolumn{2}{|c|}{ Tumor } & \multicolumn{2}{|c|}{ Liver } & \multirow[b]{2}{*}{$\begin{array}{l}\text { PET } \\
\text { results }\end{array}$} \\
\hline & & & & & & & $\begin{array}{l}\text { Mean } \\
\text { SUV }\end{array}$ & $\begin{array}{l}\text { Maximum } \\
\text { SUV }\end{array}$ & $\begin{array}{l}\text { Mean } \\
\text { SUV }\end{array}$ & $\begin{array}{l}\text { Maximum } \\
\text { SUV }\end{array}$ & \\
\hline 1 & M & 75 & $\mathrm{HCC}$ & Yes & 1 & $2-5$ & 2.5 & 2.9 & 4.7 & 5.0 & Negative \\
\hline 2 & M & 73 & $\mathrm{HCC}$ & Yes & 3 & $10-25$ & 11.1 & 14.3 & 4.4 & 4.9 & Positive \\
\hline 3 & M & 53 & $\mathrm{HCC}$ & Yes & 3 & $2-5$ & 3.8 & 4.5 & 9.0 & 11.0 & Negative \\
\hline 4 & M & 48 & $\mathrm{HCC}$ & Yes & 2 & 20 & 7.0 & 8.2 & 7.7 & 8.3 & Positive \\
\hline 5 & M & 64 & $\mathrm{HCC}$ & Yes & 2 & $2-10$ & 6.5 & 8.5 & 5.5 & 6.9 & Positive \\
\hline 6 & M & 72 & $\mathrm{HCC}$ & Yes & 2 & $2-5$ & 9.6 & 11.1 & 7.8 & 9.3 & Positive \\
\hline 7 & M & 74 & $\mathrm{HCC}$ & Yes & 2 & $2-5$ & 8.2 & 8.9 & 8.3 & 9.5 & Negative \\
\hline 8 & M & 73 & $\mathrm{CCC}$ & Yes & 2 & $30-50$ & 11.1 & 14.3 & 5.0 & 5.8 & Positive \\
\hline 9 & M & 58 & $\mathrm{HCC}$ & No & NA & NA & 6.7 & 7.9 & 4.9 & 5.9 & Positive \\
\hline 10 & M & 66 & $\mathrm{HCC}$ & Yes & NA & NA & 8.2 & 9.1 & 5.0 & 5.4 & Positive \\
\hline 11 & M & 60 & $\mathrm{HCC}$ & No & NA & NA & 6.6 & 8.1 & 5.3 & 6.5 & Positive \\
\hline 12 & M & 70 & $\mathrm{HCC}$ & Yes & 2 & 10 & 10.7 & 12.3 & 4.6 & 5.7 & Positive \\
\hline 13 & M & 77 & $\mathrm{HCC}$ & Yes & 2 & 1 & 6.4 & 7.3 & 7.2 & 8.8 & Negative \\
\hline 14 & M & 77 & $\mathrm{HCC}$ & Yes & 2 & 20 & 7.7 & 9.2 & 5.9 & 6.6 & Positive \\
\hline 15 & M & 67 & $\mathrm{CCC}$ & Yes & 2 & NA & 16.8 & 18.8 & 13.5 & 15.6 & Positive \\
\hline 16 & M & 66 & $\mathrm{HCC}$ & No & NA & NA & 7.9 & 9.0 & 9.5 & 10.2 & Negative \\
\hline 17 & M & 58 & $\mathrm{HCC}$ & Yes & 1 & 30 & 11.1 & 14.3 & 4.8 & 5.9 & Positive \\
\hline 18 & $\mathrm{~F}$ & 78 & $\mathrm{HCC}$ & No & NA & NA & 7.3 & 8.9 & 5.9 & 6.6 & Positive \\
\hline
\end{tabular}



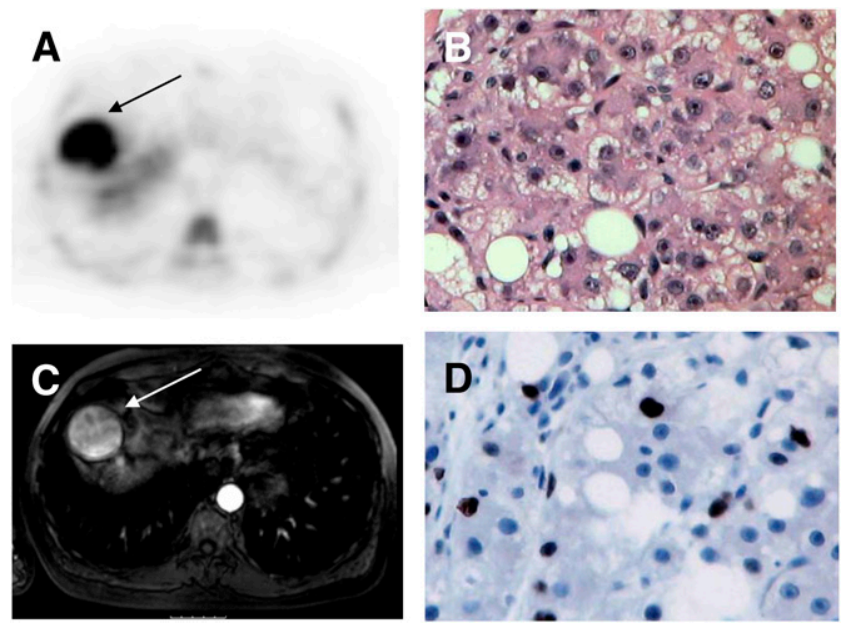

FIGURE 1. (A and C) Axial ${ }^{18}$ F-FLT PET (A) and axial MRI (C) scans of patient with multifocal HCC (patient 17). A shows increased ${ }^{18} \mathrm{~F}-\mathrm{FLT}$ uptake (black arrow) and $\mathrm{C}$ displays corresponding MRI section (white arrow). (B and D) Hematoxylin-eosin staining (magnification, $\times 20)(B)$ and immunohistochemical staining for MIB-1 (magnification, $\times 20)(D)$. MIB labeling index was $30 \%$.

range, 2.5-8.2); however, the difference did not reach statistical significance $(P=0.09)$. Mean ${ }^{18} \mathrm{~F}$-FLT uptake and maximum ${ }^{18} \mathrm{~F}$-FLT uptake in representative liver sections were higher in patients with negative results at visual analysis (7.7 and 8.9, respectively; median, 8.3 and 9.5, respectively) than in patients with positive results (5.6 and 6.5; median, 5.3 and 6.5) but did not reach statistical significance $(P=0.069$ for both mean and maximum).
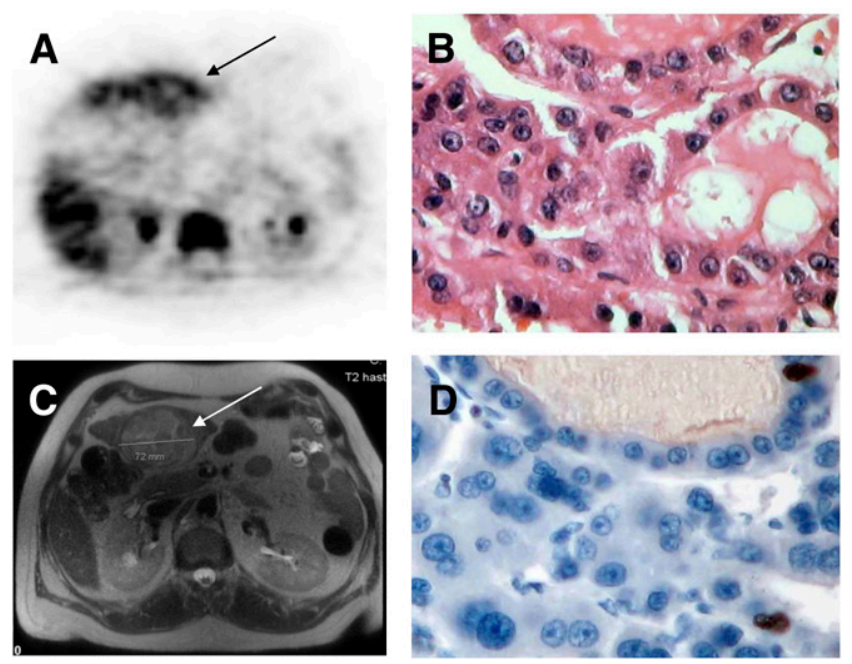

FIGURE 2. (A and C) Axial ${ }^{18} \mathrm{~F}-\mathrm{FLT}$ PET (A) and MRI (C) scans of patient with unifocal HCC (patient 3). Images show mixed uptake pattern in hepatic lesion (black arrow) (rated visually as negative for ${ }^{18} \mathrm{~F}-\mathrm{FLT}$ ). (B and D) Hematoxylineosin staining (magnification, $\times 20$ ) $(B)$ and immunohistochemical staining for MIB-1 (magnification, $\times 20$ ) (D). MIB labeling index was $2 \%-5 \%$.
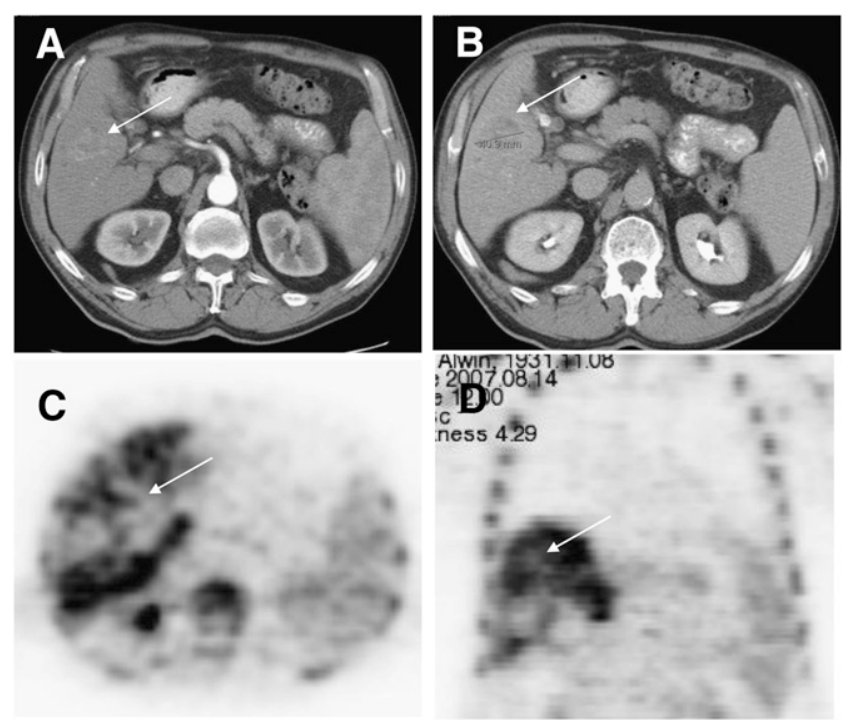

FIGURE 3. (A and B) Axial CT views of arterial-phase (A) and venous-phase (B) scans of patient with unifocal HCC (patient 1). (C and D) Axial (C) and coronal (D) views of corresponding ${ }^{18} \mathrm{~F}$-FLT PET scans. Images show decreased ${ }^{18} \mathrm{~F}-\mathrm{FLT}$ uptake in tumor lesion compared with physiologic liver uptake (arrows). MIB labeling index (not displayed) was $2 \%-5 \%$.

Both patients with CCC had ${ }^{18} \mathrm{~F}$-FLT-positive results, with mean ${ }^{18} \mathrm{~F}-\mathrm{FLT}$ uptake values of 11.1 and 16.8 ; the corresponding maximum ${ }^{18} \mathrm{~F}$-FLT uptake values were 14.3 and 18.8 .

$T / L$ Ratios. The mean $\mathrm{T} / \mathrm{L}$ ratio for mean ${ }^{18} \mathrm{~F}-\mathrm{FLT}$ uptake in HCC tumors was 1.3 (median, 1.2; range, 0.4-2.5). The T/L ratio in the subgroup of tumors with positive results at visual analysis ranged from 0.9 to 2.5 (mean, 1.6; median, 1.3) and was significantly higher than that in tumors with negative results (mean, 0.7; median, 0.8; range, 0.4-1.0) $(P=0.001)$. Some lesions showed a mixed uptake pattern (partially increased uptake and partially decreased uptake) judged as visually detectable despite uptake lower than that in the surrounding liver tissue. The T/L ratio for maximum SUVs was also higher in tumors with positive PET results (mean, 1.6; median, 1.3; range, 1.0-2.9) than in lesions with negative PET results (mean, 0.7; median, 0.8; range, 0.4-0.9) $(P<0.001)$. The T/L ratios for the 2 patients with CCC were 1.2 and 2.2 (mean SUVs) and 1.2 and 2.5 (maximum SUVs).

ROC Analysis. In the subgroup of HCC tumors, ROC analysis of the use of the T/L ratios of the mean SUVs to separate tumors with positive PET results from tumors with negative PET results revealed a T/L ratio of 1.0 to be the optimal cutoff (area under the curve, 0.985). All tumors with negative PET results (5/5) had T/L ratios of less than or equal to 1.0 (specificity, $100 \%$; 95\% CI, 48\%-100\%), and 10 of 11 lesions with positive PET results had T/L ratios of greater than 1.0 (sensitivity, 91\%; 95\% CI, $59 \%-99 \%)(P<0.001)$. ROC analysis of the use of the $\mathrm{T} / \mathrm{L}$ ratios of the maximum SUVs for tumor discrimination 
again led to an optimal cutoff of 1.0, resulting in T/L ratios of less than 1.0 for all lesions with negative PET results (specificity, 100\%; 95\% CI, 48\%-100\%) and T/L ratios of greater than or equal to 1.0 for all lesions with positive PET results (sensitivity, 100\%; 95\% CI, 72\%-100\%).

\section{Helical CT and MRI Findings for HCC Tumors}

Helical CT and MRI demonstrated an obvious tumor in all patients. The HCC was solitary in 9 patients. Two patients had a dominant mass with smaller satellite lesions. Nine of the HCC tumors were multifocal. The average diameter of the largest mass was $8.4 \mathrm{~cm}$ (range, $2.5-20 \mathrm{~cm}$ ). The dominant HCC was in the right lobe in 15 patients and in the left lobe in 3 patients. The HCC was bilobed in 2 patients. There was no evidence of calcifications, central scar, fat, or abdominal lymphadenopathy. Dilated intrahepatic bile ducts were present in one patient.

\section{Correlation of Tumor Grading and MIB Labeling Index with Visual Analysis of ${ }^{18} \mathrm{~F}-\mathrm{FLT}$ PET and Quantitative Values}

HCC tumors were graded as well differentiated, moderately differentiated, and poorly differentiated in 2, 7, and 2 patients, respectively; both CCC tumors were graded as moderately differentiated. One of the 2 well-differentiated HCC tumors had positive ${ }^{18} \mathrm{~F}$-FLT PET results. Of the 2 poorly differentiated $\mathrm{HCC}$ tumors, one had positive ${ }^{18}$ F-FLT PET results and one had negative results. The MIB labeling index was available for 11 of the $16 \mathrm{HCC}$ tumors and for one of the 2 CCC tumors. For the assessable tumor tissue, the MIB labeling index ranged between $1 \%$ and $30 \%$ (Figs. 1 and 2). Correlation analysis demonstrated a significant positive relationship between the MIB labeling index and the mean SUV $(r=0.66, P=0.02)$. All 7 tumors with an MIB labeling index of greater than or equal to $5 \%$ had positive ${ }^{18}$ F-FLT PET results, whereas 4 of the 5 tumors with an MIB labeling index of less than $5 \%$ had negative ${ }^{18}$ F-FLT PET results $(P=0.01)$. A separate analysis for HCC and CCC was not performed because of the small amount of available tumor tissue.

\section{Clinical Follow-up}

The mean follow-up after the ${ }^{18}$ F-FLT PET examination was 12.5 mo (median follow-up, $11.5 \mathrm{mo}$ ). So far, 5 patients have died and 13 are still alive. No patient was lost to follow-up. Survival analysis (Cox proportional hazards regression) was performed for initial ${ }^{18}$ F-FLT uptake (mean and maximum SUVs) and for the MIB labeling index. The hazard ratios were 1.20 for the mean SUV and 1.12 for the maximum SUV; however, because of the small number of events, the corresponding CIs (0.93-1.56 for mean SUV; 0.89-1.41 for maximum SUV) did include 1 and so failed to demonstrate statistical significance.

\section{DISCUSSION}

HCC is a relatively rare disease in Western countries but is one of the leading causes of cancer death worldwide.
Although MRI and multiphase, contrast-enhanced CT are considered to be mainstay diagnostic tests for imaging $\mathrm{HCC}$, the differential diagnosis of hepatic tumors, monitoring of the response to treatment, and the detection of recurrent disease or progression remain challenging, and further research is required. Noninvasive imaging of the proliferation fraction was recently suggested to be a suitable approach for the functional characterization of tumors and for early detection of the response to treatment. In 1998, Shields et al. reported that the thymidine analog ${ }^{18} \mathrm{~F}-$ FLT is a suitable PET radiopharmaceutical for the specific imaging of proliferation (17).

In the present in vivo study, a significant correlation of ${ }^{18} \mathrm{~F}$-FLT uptake with the proliferation fraction of HCC ( $r=$ $0.66, P=0.02$ ), as assessed by $\mathrm{Ki}-67$ immunohistochemistry, was demonstrated. Despite the recruitment so far of only a small number of patients, our data indicate that ${ }^{18} \mathrm{~F}$ FLT PET can be used to noninvasively assess the proliferative activity of HCC. This finding is consistent with reports indicating a significant correlation between ${ }^{18} \mathrm{~F}$-FLT uptake and tumor proliferation for other solid neoplasms, including non-small cell lung cancer (18), colorectal cancer (19), breast cancer (20), lymphoma (21), and sarcoma (22). To date, it remains to be determined whether specific imaging of the proliferation fraction is of any benefit for the management of proven HCC or undefined lesions clinically suspected of being HCC. However, one may speculate that focal ${ }^{18} \mathrm{~F}$-FLT uptake has the potential to identify $\mathrm{HCC}$ with higher proliferative activity and, accordingly, higher biologic aggressiveness. Whether ${ }^{18}$ F-FLT PET also can indicate which patients may benefit from radiotherapy or chemotherapy or can identify an early response to treatment remains to be determined.

Currently, MRI and multiphase CT are performed most frequently for the detection, staging, and management of hepatic tumors. The sensitivity of MRI for the detection of HCC is slightly higher than that of CT, with values of between $75 \%$ and $94 \%$ (23). The diagnosis of HCC is considered to be very likely for tumors larger than $20 \mathrm{~mm}$ and in the presence of markedly increased serum $\alpha$-fetoprotein levels $(>400 \mu \mathrm{g} / \mathrm{L})(23)$. However, for lesions smaller than $20 \mathrm{~mm}$, the specificity of $\alpha$-fetoprotein is insufficient for the diagnosis of HCC, and a biopsy is recommended. Tumors leading to biliary obstruction can be additionally imaged with magnetic resonance cholangiopancreatography or endoscopic retrograde cholangiopancreatography. The respective sensitivity and specificity for the differentiation of malignant from benign causes of biliary obstruction have been reported to be $81 \%$ and $70 \%$ for magnetic resonance cholangiopancreatography and $74 \%$ and $70 \%$ for endoscopic retrograde cholangiopancreatography (24). Delayed contrast-enhanced CT has been reported to increase tumor detection with a sensitivity of $74 \%$ and a specificity of $82 \%$.

PET with the glucose analog ${ }^{18} \mathrm{~F}$-FDG does not have a high sensitivity for the detection of HCC because highly differentiated tumors have the ability to accomplish gluco- 
neogenesis and therefore can convert FDG-6-phosphate to FDG. Consequently, the trapping of the radiopharmaceutical is reduced, resulting in a lower sensitivity. The sensitivity of ${ }^{18} \mathrm{~F}-\mathrm{FDG}$ PET can be increased to $62.5 \%$ by delaying the acquisition of PET data for 2-3 $\mathrm{h}$ (25). Therefore, the current role of ${ }^{18} \mathrm{~F}-\mathrm{FDG}$ PET in HCC applies only to the detection of extrahepatic tumor deposits in patients with ${ }^{18} \mathrm{~F}-\mathrm{FDG}$-avid primary lesions. In a pilot study (26), a change in therapeutic management in $26 \%$ of patients resulted from the detection of extrahepatic tumor deposits. However, the role of ${ }^{18}$ F-FDG PET or ${ }^{18}$ F-FDG $\mathrm{PET} / \mathrm{CT}$ in the staging and management of $\mathrm{HCC}$ needs to be evaluated in prospective studies with more patients.

Several primary tumors were also determined to have negative ${ }^{18} \mathrm{~F}$-FLT PET results by visual interpretation, resulting in a reduced sensitivity (69\%) for the detection of HCC. This finding corresponds to a reduced sensitivity of ${ }^{18}$ F-FLT PET for other solid neoplasms (lung cancer $(27,28)$, colorectal cancer (19), and pancreatic cancer (29)). In these tumor subtypes, tracer uptake markedly lower than that of the standard radiotracer ${ }^{18} \mathrm{~F}-\mathrm{FDG}$ has been observed. Therefore, no superiority of ${ }^{18} \mathrm{~F}$-FLT over ${ }^{18} \mathrm{~F}$-FDG regarding the detection and staging of cancers has been described in the latter studies. Our study indicates that the $69 \%$ sensitivity of ${ }^{18}$ F-FLT PET for the detection of HCC is not inferior to the sensitivity of ${ }^{18} \mathrm{~F}-\mathrm{FDG}$ PET $(40 \%-70 \%)$ $(10,25,30)$.

The sensitivity of PET may be increased with other innovative tracers enabling molecular tumor imaging. Recently, Ho et al. reported that HCC with negative ${ }^{18} \mathrm{~F}-\mathrm{FDG}$ PET results may be visualized with $\mathrm{PET}$ and ${ }^{11} \mathrm{C}$-acetate, which is a substrate for $\beta$-oxidation and a precursor for the biosynthesis of amino acids and fatty acids (10). The authors demonstrated ${ }^{18} \mathrm{~F}$-FDG uptake predominantly in poorly differentiated $\mathrm{HCC}$ tumors, whereas ${ }^{11} \mathrm{C}$-acetate accumulated in well-differentiated cancers. ${ }^{11} \mathrm{C}$-acetate was also specific for $\mathrm{HCC}$ and did not accumulate in metastatic liver lesions or CCC. In another study (10), the same group demonstrated high sensitivity, specificity, and accuracy for detecting extrahepatic metastases from HCC (98\%, 86\%, and 96\%, respectively). There was also a change in therapeutic management because of the detection of metastases not revealed by standard imaging modalities. ${ }^{11} \mathrm{C}$-choline has also been suggested for imaging $\mathrm{HCC}$, but the use of ${ }^{11} \mathrm{C}$-choline is hampered by physiologically high uptake of the radiotracer in the liver (31).

Hybrid scanners such as PET/CT scanners integrate morphologic information and metabolic information and allow increased sensitivity as well as specificity of noninvasive imaging. Recently, it was reported that contrastenhanced PET/CT enabled the detection of a larger number of hepatic lesions than nonenhanced PET/CT. However, because of false-negative PET findings in up to $40 \%$ of HCC tumors, ${ }^{18}$ F-FDG PET is most frequently included in a clinical work-up as a problem-solving tool rather than as a standard imaging test.
Survival analysis revealed a trend toward higher hazard ratios for mean and maximum SUVs for ${ }^{18}$ F-FLT (hazard ratios of 1.20 and 1.12 per one-unit increment, respectively), but because of the small number of events, the corresponding CIs (0.93-1.56 for mean SUV; 0.89-1.41 for maximum SUV) did include 1 and so failed to demonstrate statistical significance. Hypothetical calculations supposing 2 additional events in patients with high initial ${ }^{18} \mathrm{~F}$-FLT uptake and presuming an additional follow-up at 6 mo also did not result in statistical significance. The main limiting factor of this survival analysis was the small size of the study population, which remains an unaddressable issue because of the low incidence of HCC in Europe. Nevertheless, high initial ${ }^{18} \mathrm{~F}$-FLT uptake seems to correlate with reduced overall survival and could be an important prognostic factor if this tendency can be confirmed in a larger prospective trial.

Several limitations must be considered in applying our results to the clinical situation. First, the number of included patients with HCC (18) was small, and the high correlation of tracer uptake with the proliferation fraction may not carry over into a larger series. Second, lower rates of detection of liver lesions smaller than $10 \mathrm{~mm}$ is a wellknown limitation of PET. The reduced sensitivity for small liver lesions is caused predominantly by partial-volume effects and respiratory motion artifacts. Because of the increased background activity of ${ }^{18} \mathrm{~F}-\mathrm{FLT}$, presumably resulting from the glucuronidation of ${ }^{18}$ F-FLT in the liver, sensitivity for the detection of small lesions may also be reduced with ${ }^{18} \mathrm{~F}$-FLT PET. Third, the study population did not cover all histologic subtypes of HCC, and the rate of false-negative findings may increase if patients with highly differentiated cancers are imaged with ${ }^{18}$ F-FLT PET.

\section{CONCLUSION}

The present in vivo study indicates that imaging the proliferative activity of HCC with the in vivo proliferation marker ${ }^{18} \mathrm{~F}$-FLT is feasible. The sensitivity of $69 \%$ for detecting $\mathrm{HCC}$ is lower than the corresponding values published for CT and MRI. However, the approach of molecular imaging of proliferation may, in combination with anatomically based imaging modalities, increase specificity and aid in the differential diagnosis of benign and malignant tumors. The observed association between high initial ${ }^{18} \mathrm{~F}$-FLT uptake and reduced overall survival needs to be confirmed in a larger prospective trial, evaluating the potential of risk stratification and treatment selection using ${ }^{18}$ F-FLT PET.

\section{ACKNOWLEDGMENTS}

We appreciate the excellent contributions made by our colleagues Petra Watzlowik, Karin Kantke, and Michael Herz and the great support provided by Christine Praus, our students Barbara Numberger and Daniela Zwiesler, and our 
technical staff members Brigitte Dzewas and Coletta Kruschke.

\section{REFERENCES}

1. Bosch FX, Ribes J, Diaz M, Cleries R. Primary liver cancer: worldwide incidence and trends. Gastroenterology. 2004;127(suppl 1):S5-S16.

2. Davila JA, Morgan RO, Shaib Y, McGlynn KA, El-Serag HB. Hepatitis C infection and the increasing incidence of hepatocellular carcinoma: a populationbased study. Gastroenterology. 2004;127:1372-1380.

3. Bralet MP, Regimbeau JM, Pineau P, et al. Hepatocellular carcinoma occurring in nonfibrotic liver: epidemiologic and histopathologic analysis of 80 French cases. Hepatology. 2000;32:200-204.

4. Llovet JM. Updated treatment approach to hepatocellular carcinoma. $J$ Gastroenterol. 2005;40:225-235.

5. Saar B, Kellner-Weldon F. Radiological diagnosis of hepatocellular carcinoma. Liver Int. 2008;28:189-199.

6. Chapman WC, Majella Doyle MB, Stuart JE, et al. Outcomes of neoadjuvant transarterial chemoembolization to downstage hepatocellular carcinoma before liver transplantation. Ann Surg. 2008;248:617-625.

7. Llovet JM, Bruix J. Systematic review of randomized trials for unresectable hepatocellular carcinoma: chemoembolization improves survival. Hepatology. 2003;37:429-442.

8. Khan MA, Combs CS, Brunt EM, et al. Positron emission tomography scanning in the evaluation of hepatocellular carcinoma. J Hepatol. 2000;32:792-797.

9. Trojan J, Schroeder O, Raedle J, et al. Fluorine-18 FDG positron emission tomography for imaging of hepatocellular carcinoma. Am J Gastroenterol. 1999;94:3314-3319.

10. Ho CL, Yu SC, Yeung DW. ${ }^{11} \mathrm{C}$-acetate PET imaging in hepatocellular carcinoma and other liver masses. J Nucl Med. 2003;44:213-221.

11. Buck AK, Halter $\mathrm{G}$, Schirrmeister $\mathrm{H}$, et al. Imaging proliferation in lung tumors with PET: ${ }^{18}$ F-FLT versus ${ }^{18}$ F-FDG. $J$ Nucl Med. 2003;44:1426-1431.

12. Wagner M, Seitz U, Buck A, et al. $3^{\prime}-\left[{ }^{18} \mathrm{~F}\right]$ fluoro- $3^{\prime}$-deoxythymidine $\left(\left[{ }^{18} \mathrm{~F}\right]\right.$-FLT) as positron emission tomography tracer for imaging proliferation in a murine Bcell lymphoma model and in the human disease. Cancer Res. 2003;63:26812687.

13. Bruix J, Sherman M. Management of hepatocellular carcinoma. Hepatology. 2005;42:1208-1236.

14. Machulla HJ, Blocher A, Kuntzsch M, Grierson JR. Simplified labeling approach for synthesizing $3^{\prime}$-deoxy-3'-[ $\left.{ }^{18} \mathrm{~F}\right]$ fluorothymidine ( $\left.\left[{ }^{18} \mathrm{~F}\right] \mathrm{FLT}\right)$. J Radioanal Nucl Chem. 2000;24:843-846.

15. Herrmann K, Wieder HA, Buck AK, et al. Early response assessment using 3'deoxy- $3^{\prime}-\left[{ }^{18} \mathrm{~F}\right]$ fluorothymidine-positron emission tomography in high-grade non-Hodgkin's lymphoma. Clin Cancer Res. 2007;13:3552-3558.
16. Weber WA, Ziegler SI, Thodtmann R, Hanauske AR, Schwaiger M. Reproducibility of metabolic measurements in malignant tumors using FDG PET. J Nucl Med. 1999;40:1771-1777.

17. Shields AF, Grierson JR, Dohmen BM, et al. Imaging proliferation in vivo with [F-18]FLT and positron emission tomography. Nat Med. 1998;4:1334-1336.

18. Buck AK, Schirrmeister H, Hetzel M, et al. 3-deoxy-3- $\left[{ }^{18} \mathrm{~F}\right]$ fluorothymidinepositron emission tomography for noninvasive assessment of proliferation in pulmonary nodules. Cancer Res. 2002;62:3331-3334.

19. Francis DL, Freeman A, Visvikis D, et al. In vivo imaging of cellular proliferation in colorectal cancer using positron emission tomography. Gut. 2003;52:1602-1606.

20. Kenny LM, Vigushin DM, Al-Nahhas A, et al. Quantification of cellular proliferation in tumor and normal tissues of patients with breast cancer by $\left[{ }^{18} \mathrm{~F}\right]$ fluorothymidine-positron emission tomography imaging: evaluation of analytical methods. Cancer Res. 2005;65:10104-10112.

21. Buck AK, Bommer M, Stilgenbauer S, et al. Molecular imaging of proliferation in malignant lymphoma. Cancer Res. 2006;66:11055-11061.

22. Buck AK, Herrmann K, Büschenfelde CM, et al. Imaging bone and soft tissue tumors with the proliferation marker $\left[{ }^{18} \mathrm{~F}\right]$ fluorodeoxythymidine. Clin Cancer Res. 2008;14:2970-2977.

23. Fung KT, Li FT, Raimondo ML, et al. Systematic review of radiological imaging for hepatocellular carcinoma in cirrhotic patients. Br J Radiol. 2004;77:633-640.

24. Park MS, Kim TK, Kim KW, et al. Differentiation of extrahepatic bile duct cholangiocarcinoma from benign stricture: findings at MRCP versus ERCP. Radiology. 2004;233:234-240.

25. Lin WY, Tsai SC, Hung GU. Value of delayed ${ }^{18}$ F-FDG-PET imaging in the detection of hepatocellular carcinoma. Nucl Med Commun. 2005;26:315-321.

26. Sugiyama M, Sakahara H, Torizuka T, et al. ${ }^{18} \mathrm{~F}$-FDG PET in the detection of extrahepatic metastases from hepatocellular carcinoma. $J$ Gastroenterol. 2004;39:961-968.

27. Buck AK, Hetzel M, Schirrmeister H, et al. Clinical relevance of imaging proliferative activity in lung nodules. Eur J Nucl Med Mol Imaging. 2005;32: 525-533.

28. Yap CS, Czernin J, Fishbein MC, et al. Evaluation of thoracic tumors with ${ }^{18} \mathrm{~F}$ fluorothymidine and ${ }^{18} \mathrm{~F}$-fluorodeoxyglucose positron emission tomography. Chest. 2006;129:393-401.

29. Herrmann K, Eckel F, Schmidt S, et al. In vivo characterization of proliferation for discriminating cancer from pancreatic pseudotumors. J Nucl Med. 2008; 49:1437-1444.

30. Park JW, Kim JH, Kim SK, et al. A prospective evaluation of ${ }^{18} \mathrm{~F}-\mathrm{FDG}$ and ${ }^{11} \mathrm{C}$ acetate PET/CT for detection of primary and metastatic hepatocellular carcinoma. J Nucl Med. 2008;49:1912-1921.

31. Yamamoto Y, Nishiyama Y, Kameyama R, et al. Detection of hepatocellular carcinoma using ${ }^{11} \mathrm{C}$-choline PET: comparison with ${ }^{18} \mathrm{~F}-\mathrm{FDG}$ PET. J Nucl Med. 2008;49:1245-1248. 